\title{
Use of MRI Apparent Diffusion Coefficient to Noninvasively Differentiate between the Histologic Grades of Meningioma
}

\author{
Neethu P. M. ${ }^{1}$, Saanida M. P.², Gomathi Subramaniam³ ${ }^{3}$ Devarajan E.4, Naufal P.5 , Juvaina P.6
}

\begin{abstract}
${ }^{1}$ Department of Radiodiagnosis, Government Medical College, Kozhikode, Kerala, India. ${ }^{2}$ Department of Radiodiagnosis, Government Medical College, Kozhikode, Kerala, India. ${ }^{3}$ Department of Radiodiagnosis, Government Medical College, Kozhikode, Kerala, India. ${ }^{4}$ Department of Radiodiagnosis, Government Medical College, Kozhikode, Kerala, India. ${ }^{5}$ Department of Radiodiagnosis, Government Medical College, Kozhikode, Kerala, India. ${ }^{6}$ Department of Radiodiagnosis, Government Medical College, Kozhikode, Kerala, India.
\end{abstract}

\section{ABSTRACT}

\section{BACKGROUND}

It is well known in the literature that Apparent Diffusion Coefficient (ADC) obtained during diffusion-weighted MRI of brain is sensitive in detecting and differentiating low-grade and aggressive meningiomas. However, other studies establish no correlation between mean-ADC and ultimate classification as benign, atypical or malignant. We wanted to assess the correlation between, A. mean of MRI Apparent Diffusion Coefficient (mean-ADC) and B. meningioma biomarker Ki-67 proliferation index, in cases of both low-grade and aggressive meningioma. We also wanted to assess the sensitivity of mean-ADC for diagnosing an aggressive meningioma.

\section{METHODS}

We analysed 66 surgically treated meningioma patients with complete histopathology report (HPR) in Government Medical College, Kozhikode during the period of study (Nov-2017-Oct-2018), also having a pre-operative mean-ADC value during Diffusion Weighted Imaging (DWI) investigation at the same centre. For the latter, a standard MRI brain protocol including diffusion imaging was conducted and ADC map was generated. Regions of interest (ROIs) were manually drawn within the tumour on ADC map and mean-ADC values were measured.

\section{RESULTS}

Using Pearson correlation coefficient, we found a significant negative correlation between Ki-67 proliferation index and mean-ADC in meningioma cases. We also calculate $0.79 \times 10^{-3} \mathrm{~mm}^{2} / \mathrm{s}$ being a reasonable mean-ADC cut-off value due to its sensitivity of $84 \%$ and specificity of $77 \%$ in differentiating between typical and atypical meningiomas.

\section{CONCLUSIONS}

The non-invasive calculation of mean-ADC is a valid diagnostic tool. Further, mean ADC can be used as a good test to differentiate typical and atypical meningiomas. The latter is a particularly valid conclusion, since there were few results from conventional MRI studies to differentiate between various typical and atypical meningiomas.

\section{KEY WORDS}

MRI Apparent Diffusion Coefficient (ADC), Meningioma, WHO I, II and III Meningiomas, Typical and Atypical Meningiomas, Low-Grade and High-Grade Meningiomas

\author{
Corresponding Author: \\ Dr. Saanida M. P., \\ Assistant Professor, \\ Department of Radiodiagnosis, \\ Government Medical College, \\ Kozhikode-673006, Kerala, India. \\ E-mail: saanida.manthampally@gmail.com
}

DOI: $10.14260 /$ jemds/2020/369

Financial or Other Competing Interests: None.

How to Cite This Article:

Neethu PM, Saanida MP, Subramaniam G, et al. Use of MRI apparent diffusion coefficient to noninvasively differentiate between the histologic grades of meningioma. J. Evolution Med. Dent. Sci. 2020;9(22):1679-1683, DOI:
Submission 17-02-2020,

Peer Review 06-05-2020,

Acceptance 13-05-2020,

Published 01-06-2020. 


\section{BACKGROUND}

It is well known in the literature,[1][2] that Apparent Diffusion Coefficient (ADC) obtained during diffusion-weighted MRI of brain is sensitive in detecting and differentiating low-grade and aggressive meningiomas. However, other studies such as [3] establish no correlation between mean-ADC and ultimate classification as benign, atypical or malignant. Our study establishes relevance of this finding to a population of North Kerala, with aim to propose inclusion of mean-ADC obtained from Diffusion-Weighted MRI sequence in protocol of the centre.

\section{Objectives}

- To compare ADC with post-operative biomarker Ki-67 in predicting grade of meningioma

- To evaluate ADC as distinguishing between typical (benign, WHO grade I) and atypical/malignant (WHO grades II and III) meningioma.

MRI Diffusion Weighted Imaging (DWI), and the inferred metric mean-ADC, are superior in diagnosing grade of meningioma (benign, atypical, and malignant) since most meningiomas do not restrict on DWI. However, only gross impression of ADC and cellularity obtained from DWI was part of the protocol for such cases in centres such as our institution Govt Medical College, Kozhikode, Kerala, India there was no reasonable mean-ADC value around which to propose an impression of whether diagnosed meningioma was benign, atypical or malignant.

Such studies are relevant because Diffusion-weighted MRI and ADC analysis would aid in early and pre-operative diagnosis of meningioma being atypical or malignant. Thus, good diagnostic ability would help in an informed prognosis for patient's surgery. Mean-ADC values are typically lower than normal brain values for malignant meningiomas and these are hyperintense on diffusion-weighted images, while being hypointense on ADC maps. Meningiomas which are benign have mean-ADC values higher than normal brain values. On diffusion-weighted images and ADC maps, these are likely to be isointense. In many cases, benign meningiomas could have very high mean-ADC values, i.e. bright signal on ADC maps, and distinct post-operative findings such as microcysts or necrotic infarcts often linked to increased water content.

Meningiomas are mostly asymptomatic and comprise a fifth of all intracranial tumours. However, atypical and malignant meningiomas account for $10 \%$ of all meningiomas, and are more prone to recurrence and aggressive growth, which in turn causes patient morbidity and mortality despite an initial good prognosis. It would thus be useful to distinguish among benign, malignant, and atypical meningiomas before surgical resection.

Some justification is available by examining the postoperative histopathologic findings of atypical and malignant meningiomas, which correlate well with their appearance on diagnostic diffusion-weighted images and mean-ADC values. The properties of hypercellularity, multifocal areas of necrosis, brain invasion, numerous abnormal mitoses, or cytologic pleomorphism observed in these meningiomas correspond to less extracellular water and space, which reduces mean-ADC value. Primary brain neoplasms, of which meningioma's are a major family, once treated show an increase in extracellular water and space due to cell lysis thereby causing an increase in the diffusion constant of mean-ADC.

\section{METHODS}

\section{Study Design}

Cross-sectional study.

\section{Sample Size}

Using convenience sampling, we calculate total sample size required for this correlation study. Total sample size, $\mathrm{N}=$ $(1 / c) *[z 1+z 2]^{2}+3=66$, given the standard normal deviate for type- 1 error, i.e. $\mathrm{z} 1=1.96$ for $5 \%$ level of significance, and standard normal deviate for power of study, i.e. $\mathrm{z} 2=0.842$, while $c=0.25^{*} \ln [(1+r) /(1-r)]=0.125$. In this we use $r=0.52$, from a previous review of such studies [4] treating it as a pilot study. Thus, the total sample size we obtain is $\mathrm{N}=66$.

\section{Study Setting}

Patients 66 in number where A. DWI MRI diagnosis of meningioma and mean-ADC calculated pre-operatively, followed by B. surgical resection and complete histopathology report (HPR) as obtained from Departments of Radio diagnosis and Pathology, Government Medical College Kozhikode (also known as Calicut), Kerala, India, included in the study after passing other relevant inclusion criteria.

\section{Study Duration}

12 months from Nov. 2017 to Oct. 2018.

\begin{abstract}
Study Method
After collecting basic data and patient information in a proforma, all 66 subjects were evaluated on $1.5 \mathrm{~T}$ clinical Signa scanners (GE Healthcare) with an 8-channel head coil. Protocol was: unenhanced and contrast enhanced spin echo T1 weighted imaging, fast spin echo T2 weighted imaging, FLAIR and DWI performed in axial planes with slide thickness of $5 \mathrm{~mm}$, inter-slice gap of $1 \mathrm{~mm}$ and FOV $22 \mathrm{~cm}$. Next, DWI was performed with a single shot spin-echo echo planar imaging sequence using a TR/TE, 5000/80 matrix 96 x 128, 1 signal acquired while $b$ values were 0 and $1000 \mathrm{~s} / \mathrm{mm}^{2}$ with 3 gradient directions. Trace ADC maps was generated on an ADW workstation v 4.6 (GE Healthcare). Regions of interest (ROIs) were manually drawn within the tumour on ADC maps and mean ADC values measured. As regards Pathology, histology was available in all 66 patients after surgical resection. Meningiomas were classified according to WHO 2016 classification. Tumour proliferation indices were reported as the percentage of tumour cell nuclei labelling
\end{abstract}


with Ki67 (clone MIB-1) monoclonal antibody in formalin fixed paraffin embedded tissue section.

\section{Inclusion Criteria}

Surgically treated meningioma cases were enrolled in this study, after obtaining their informed consent and Institutional Ethics Committee approval (ref. GMCKKD/RP2017/EC/175) earlier from Government Medical College, Kozhikode, and Kerala, India. Enrolled patients also provided their complete pathologic report and pre-operative mean-ADC values.

\section{Exclusion Criteria}

- Surgically treated meningioma cases without complete pathologic report/Ki67 proliferation index.

- Surgically treated meningioma cases where MRI investigation was done outside our institution.

- Surgically treated meningioma less than $1 \mathrm{~cm}$ in diameter not supporting a reliable mean-ADC.

- Surgically treated highly calcified meningioma where reliable mean-ADC is difficult to find.

\section{Statistical Analysis}

From the data obtained, statistical analyses were done using SPSS software and Microsoft Excel. Correlation coefficients was calculated for mean-ADC and Ki67 proliferation index values from all meningiomas using linear regression. Correlation analysis was performed with Pearson correlation as the metric. A value of $\mathrm{p}<0.05$ was considered statistically significant. Comparison of mean-ADC and Ki67 proliferation index values were made between low grade and aggressive meningiomas using an independent unpaired student $\mathrm{T}$ test. The sensitivity of mean-ADC for diagnosing aggressive meningioma was calculated using ROC curve analysis.

\section{RESULTS}

\begin{tabular}{|c|c|c|c|}
\hline $\begin{array}{l}\text { ADC Measured in } \\
\text { Meningioma Type }\end{array}$ & $\mathbf{N}$ & $\begin{array}{c}\text { Mean-ADC } \\
\text { (unit is } \mathbf{1 0}^{-3} \mathrm{~mm}^{2} / \mathrm{s} \text { ) }\end{array}$ & S.D. \\
\hline Typical & 53 & 0.8579 & 0.11033 \\
\hline Atypical & 13 & 0.7108 & 0.10420 \\
\hline
\end{tabular}

There was significant difference in ADC values between typical and atypical meningioma cases with a mean of $0.85 \mathrm{x}$ $10^{-3} \mathrm{~mm}^{2} / \mathrm{s}$ for typical and $0.71 \times 10^{-3} \mathrm{~mm}^{2} / \mathrm{s}$ for atypical meningioma cases, with supporting $P$ value of 0.001 .

\begin{tabular}{|c|c|c|c|}
\hline \multicolumn{2}{|c|}{$\begin{array}{l}\text { Finding Correlation of } \\
\text { Mean-ADC with Ki-67 }\end{array}$} & $\begin{array}{l}\text { Statistical } \\
\text { Measures }\end{array}$ & Remarks \\
\hline Ki-67 biomarker & $\begin{array}{c}\text { Pearson Correlation } \\
\text { Sigma (2-tailed) } \\
\text { N }\end{array}$ & $\begin{array}{l}1 \\
66\end{array}$ & Base quantity \\
\hline \multirow{2}{*}{$\begin{array}{l}\text { Pre-operative Mean- } \\
\text { ADC measurement }\end{array}$} & $\begin{array}{l}\text { Pearson Correlation } \\
\text { Sigma (2-tailed) }\end{array}$ & $\begin{array}{c}-0.743 \\
.00\end{array}$ & $\begin{array}{l}\text { Significant at } \\
\mathrm{p}=0.002\end{array}$ \\
\hline & $\mathrm{N}$ & 66 & $\begin{array}{c}\text { R-squared value } \\
0.552\end{array}$ \\
\hline
\end{tabular}

Note that above comparison conveys more information than 4 other, earlier, studies: A. 3 other studies, found negative correlation but with lower R-squared values of 0.34 , $0.297,0.372$, respectively, implying lower confidence, B. 1 study found no significant correlation between post-operative $\mathrm{Ki}-67$ values and pre-operative mean-ADC values.

\begin{tabular}{|cccc|}
\hline $\begin{array}{c}\text { Area under } \\
\text { ROC }\end{array}$ & $\begin{array}{c}\text { Standard } \\
\text { Error }\end{array}$ & $\begin{array}{c}\text { Asymptotic } \\
\text { Sigma }\end{array}$ & $\begin{array}{c}\text { Asymptotic 95\% } \\
\text { Confidence Interval } \\
\text { Lower Bound }\end{array}$ \\
0.843 & .062 & .000 & 0.721 \\
\hline \multicolumn{4}{c|}{ Table 3. Area under ROC Curve Findings } \\
\hline
\end{tabular}

ROC curve and area under the ROC curve were analysed and found to have value 0.843 . This signifies a high-accuracy classifier and suggests that mean-ADC can be used as a reasonably good pre-operative diagnostic tool for differentiating atypical and typical meningioma.

\begin{tabular}{|ccccc|}
\hline Investigation & Enhancement Type & Typical & Atypical & Total \\
Contrast MRI & Homogenous & 27 & 7 & 34 \\
Total & Heterogenous & 26 & 6 & 32 \\
\hline \multicolumn{4}{|c}{ Table 4. Distribution of Contrast Enhancement in } \\
Typical and Atypical Meningiomas \\
\hline \multicolumn{4}{c}{} \\
\end{tabular}

In our study we evaluated post contrast enhancement characteristics and no significant difference in contrast enhancement characteristics among typical and atypical meningiomas was made out.

\begin{tabular}{|ccccc|}
\hline Investigation & Type of Meningiomas & $\mathbf{N}$ & Mean & S.D. \\
Ki-67 Proliferation & Typical & 53 & 3.21 & 3.713 \\
Index & Atypical & 13 & 13.69 & 7.099 \\
\hline \multicolumn{4}{c}{ Table 5. Ki-67 Proliferation Index in } \\
& Typical and Atypical Meningiomas \\
\hline
\end{tabular}

However, it is not surprising that post-operative HPR indicated that Ki-67 biomarker level was well correlated with findings of atypical or malignant meningioma in the sample. There was significant difference in Ki67 proliferation index between typical and atypical meningioma cases with a mean of 3.21 for typical and 13.69 for atypical meningioma cases, with $p$ value 0.001 .

\section{DISCUSSION}

Most of the meningiomas are slow-growing tumours with surgery being the treatment of choice with good prognosis. However, a small percentage of meningiomas show aggressive behaviour with increased risk of recurrence following treatment.(5) Histological analysis was not always adequate in explaining the biological behaviour of the meningiomas and Ki-67 proliferation index was introduced along with histology to better assess the same and to further grade the meningiomas. ${ }^{(6)} \mathrm{Ki}-67$ proliferation index is a good marker to predict the biological behaviour of meningiomas and, more importantly, to assess the risk of recurrence which in turn has a key role in patient management and prognosis.(6)

A non-invasive marker of Ki-67 proliferation index would thus help in a big way in managing meningioma cases and will help to individualize patient management. The last 2 decades have seen numerous studies which evaluated the 
role of MRI in predicting the histological subtype and to further grade the meningiomas.(1),(7),(8) Early studies evaluated the role of conventional MRI, signal characteristics in T1W and T2W images to identify the subtypes of typical meningioma. (8) These studies were conducted in a small number of meningioma cases and no signal characteristics were found to be specific for any subtype except angiomatous and secretory subtypes which showed T2W-hyperintense signals. However, these meningioma cases were rare in most of the studies.

With the introduction of newer physiology-based MRI techniques, various authors evaluated the role of these newer techniques in assessing meningioma subtypes and grades non-invasively. Among these mean-ADC values and perfusion imaging were the parameters that appear to be most evaluated to assess meningioma grades. Correlation of meanADC values with Ki-67 proliferation index was evaluated in multiple studies, but no consistent results were obtained.(7)(10) In our study, we tried to evaluate the correlation between ADC mean and Ki67 proliferation index among typical and atypical meningiomas. We further propose a reasonable mean-ADC threshold to classify meningiomas as typical or atypical, and to help in patient management at our centre viz. Government Medical College, Kozhikode, Kerala, India. Note that in our study, there was no case of anaplastic or malignant meningioma in the category of atypical meningioma.

In the study(8) on T2-weighted images, hypointense meningiomas were mainly fibroblastic and hyperintense tumours were mainly syncytial and angioblastic, and partly transitional. Isointense tumours were mainly transitional and partly fibroblastic and syncytial. The authors conclude that the signal intensity of the MRI may be useful in the preoperative characterization of intracranial meningiomas. T1-weighted images may predict the presence of cysts and intra-tumour blood vessels, whilst T2-weighted images can give information about histological subtype and vascularity. In our study, we evaluated the T2W signal characteristics of 66 meningiomas. Out of the 66 meningioma cases, most of the meningiomas (69.7\%) showed isointense signals compared to brain parenchyma in $\mathrm{T} 2 \mathrm{~W}$ images, and an equal $(15.2 \%$ each) number of meningiomas showed hypointense and hyperintense signals in T2W images. In this study, most of the cases were typical meningioma, and the most common histological variants were meningothelial, transitional and fibrous variants. These three histological variants predominantly showed isointense signals in $\mathrm{T} 2 \mathrm{~W}$ images. However, among the T2 hypointense tumours, there was equal distribution of fibrous and transitional variants. Hence, we conclude that $\mathrm{T} 2 \mathrm{~W}$ signal characteristics are less reliable in predicting the histological subtype. Atypical meningiomas also did not show any specific signal characters to aid in their diagnosis. In this study, we had 2 cases of angiomatous variants and they showed $\mathrm{T} 2 \mathrm{~W}$-hyperintense signals however they may be considered rare variants.

In the study(1) the authors evaluated morphological features in typical and atypical meningiomas and found out that irregular margins, bone erosion and peritumoral oedema were significantly more common in atypical meningiomas. Various other studies concluded shape was the only significant morphological feature that helped in differentiating atypical meningioma from typical meningioma. In our study, we evaluated the distribution of the pattern of contrast enhancement and peritumoral oedema among the meningiomas. Contrast enhancement had a similar distribution in both typical and atypical meningiomas, however, it is also the case that peritumoral oedema was more commonly visualized in atypical meningiomas but with lower specificity ( $44 \%$ vs $23 \%$ ).

\section{CONCLUSIONS}

Our study shows a statistically significant inverse correlation between mean-ADC and Ki-67 proliferation index values in typical and atypical meningiomas and a statistically significant difference of ADC values between low-grade and more aggressive subtypes. This has implications for prognosis and management. Specifically, a more watchful follow-up for a less favourable course and tumour recurrence would be prudent in low-grade cases with lower mean-ADC values. Thus, low mean-ADC values correlate with high Ki-67 proliferation index and help differentiate low-grade from more aggressive meningiomas and could be an important marker for treatment planning.

\section{Limitations}

Sample size in this study, while justified using typical samplesize formulas, is small. With similar sample sizes, previous studies have showed controversial results. However, we need a multi-centre study with a large sample size to better assess the relationship between mean-ADC and prediction of meningioma type. There was no case of anaplastic meningioma in our study, and the bulk of the study group were typical meningiomas, as is the case among regular occurrences. In our study, we did not follow up these patients, on a long-term basis after surgical resection, to assess whether the prognosis in-fact materialized. Finally, in this study, metrics such as min-ADC of the tumour or NADC studied in a few other investigations - were not evaluated.

\section{REFERENCES}

[1] Nagar VA, Ye JR, Ng WH, et al. Diffusion-weighted MR imaging: diagnosing atypical or malignant meningiomas and detecting tumor dedifferentiation. American Journal of Neuroradiology 2008;29(6):1147-52.

[2] Tang Y, Dundamadappa SK, Thangasamy S, et al. Correlation of apparent diffusion coefficient with Ki-67 proliferation index in grading meningioma. American Journal of Roentgenoly 2014;202(6):1303-8.

[3] Sanverdi SE, Ozgen B, Oguz KK, et al. Is diffusionweighted imaging useful in grading and differentiating histopathological subtypes of meningiomas? Eur J Radiol 2012;81(9):2389-95.

[4] Shibuya M. Pathology and molecular genetics of meningioma: recent advances. Neurologia Medica Chirurgica (Tokyo) 2015;55(1):14-27. 
[5] Louis DN, Perry A, Reifenberger G, et al. The 2016 World Health Organization Classification of Tumors of the Central Nervous System: a summary. Acta Neuropathologica (Berlin) 2016;131(6):803-20.

[6] Bohra H, Rathi KR, Dudani S, et al. The study of MIB-1 LI and $\mathrm{CD} 34$ as a marker of proliferative activity and angiogenesis in different grades of meningioma. Journal of Clinical Diagnosis Research 2016;10(8):EC14-7.

[7] Surov A, Gottschling S, Mawrin C, et al. Diffusionweighted imaging in meningioma: prediction of tumor grade and association with histopathological parameters. Translational Oncology 2015;8(6):517-23.
[8] Maiuri F, İaconetta $G$, de Divitiis 0 , et al. Intracranial meningiomas: correlations between MR imaging and histology. European Journal of Radiology 1999;31(1):6975.

[9] Ginat DT, Mangla R, Yeaney G, et al. Correlation of diffusion and perfusion MRI with Ki-67 in high-grade meningiomas. American Journal of Roentgenology 2010;195(6):1391-5.

[10] Baskan O, Silav G, Bolukbasi FH, et al. Relation of apparent diffusion coefficient with Ki-67 proliferation index in meningiomas. British Journal of Radiology 2016;89(1057):20140842. 